\title{
Earth like planets albedo variations versus continental landmass distribution
}

\author{
Esther Sanromá ${ }^{1}$ and Enric Pallé ${ }^{1}$ \\ ${ }^{1}$ Instituto de Astrofísica de Canarias (IAC), \\ Vía Láctea s/n 38200, La Laguna, Spain \\ email: mesr@iac.es
}

\begin{abstract}
By making use of real information about the continental and oceanic surface distribution of the Earth, and cloudiness data from the International Satellite Cloud Climatology Project (ISCCP), we have studied the large-scale cloudiness behavior according to latitude and surface types (ice, water, vegetation and desert). These empirical relationships are used here to reconstruct the possible cloud distribution of historical epochs of the Earth history such as Late Cretaceous (90 My ago) and Late Triasic (230 My ago) when the landmass distribution was different. This information can be used to simulate the photometric variability of these planets according to their different geographical distribution.
\end{abstract}

Keywords. astrobiology, atmospheric effects, Earth, planets and satellites: general

\section{Introduction}

Over the past few years, advances in astronomy have enabled us to discover more and more planets orbiting around stars other than the Sun, making this planet search one of the most active and exciting fields in astrophysics. It is only a matter of time before we could detect Earth-like planets.

The exploration of our own planet will provide a useful tool to interpret observations and characterize such terrestrial worlds. Because of that, in this poster we present a study about clouds and their effects on the Earth's reflectance. As it is reasonable to expect that the future observed population of planets in the galaxy will exhibit a wide range of planet types and evolutionary stages, we have studied this cloud-reflectance relation not only for the Earth at present day, but also for past epochs in the history of our planet such as the Earth 90 and 230 My ago.

\section{Data analysis}

To carry out our study, we have made use of real satellite data of the Earth's total cloud amount from the ISCCP (Rossow et al. 1996), with the aim of finding a way to modelate the distribution of clouds over the Earth's surface. By making use of real information about the surface properties of the Earth, i.e., the location of deserts, forests and oceans, we have classified the averaged cloud conditions according to different surface types, obtaining empirical relationships between the amount of clouds, surface type and latitude. We have found that these derived cloudiness functions have a very particular shape which is different for each surface type. Therefore, global cloudiness distribution could be empirically traced depending on the latitude and surface type.

These empirical relations have been used to reconstruct the possible cloud distribution of hypothetical planets, with very idealized surface properties (Sanromá et al. in preparation), and also the possible cloud distribution of past epochs of the Earth history such 


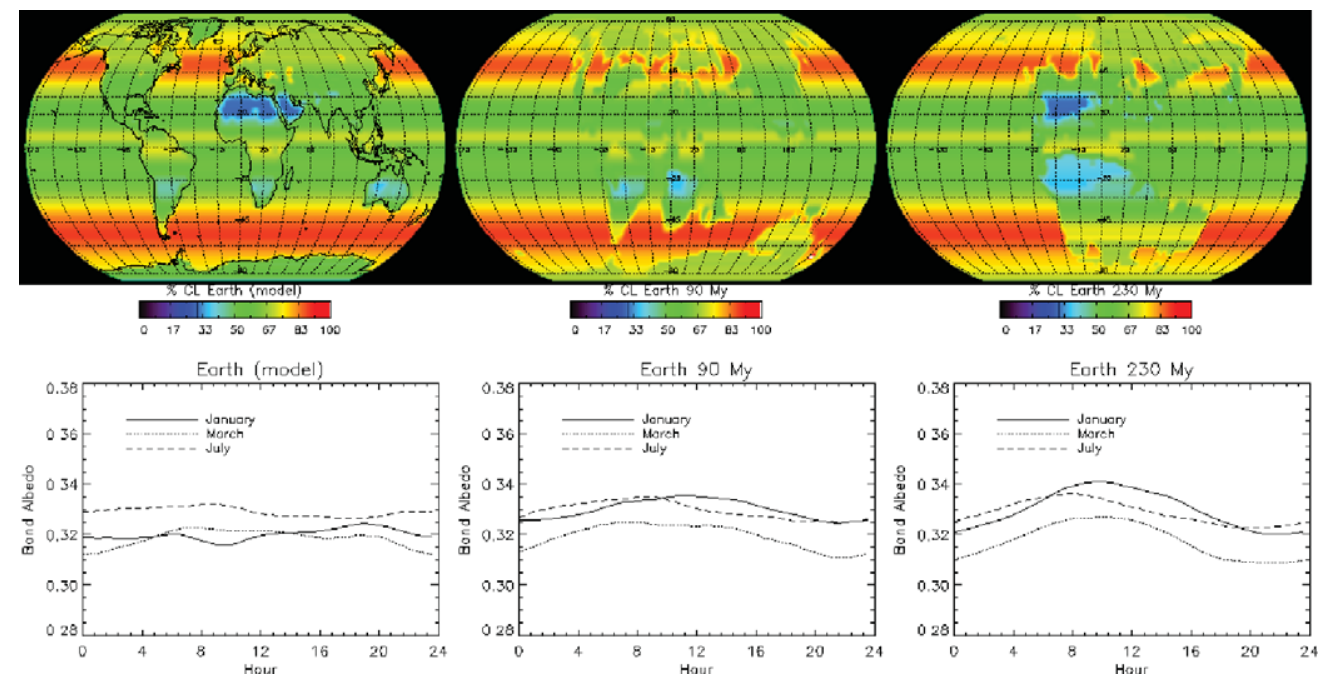

Figure 1. Top figures illustrate the reconstructed cloudiness of the Earth at present, 230 and $90 \mathrm{My}$ ago. Bottom figures show the corresponding Bond Albedo 24 hour variability for three different months.

as Late Cretaceous and Late Triasic. To check our model, we have also reconstructed the cloudiness of the Earth at present obtaining that it reproduces the general features of the Earth's cloudiness distribution.

With these reconstructed cloud maps and using a simple Earth reflectance model (Pallé et al. 2003; Pallé et al. 2008) we have computed the first-order globally-integrated photometric variability of each epoch. Here we only show the results obtained for the Earth 230, 90 My ago, and at present (Fig. 1). Our results show that the less fractioned the planet's continental surface is, the larger the amplitude of variations is. By comparing the results obtained for the Earth, one can see that historical epochs of the Earth history present larger amplitude of daily variations in reflected light and larger mean albedo than at present.

\section{References}

Pallé, E., Ford, E. B., Seager, S., Montañés-Rodríguez, P., \& Vazquez, M. 2008 ApJ, Vol. 676, 1319

Pallé, E., Goode, P. R., Yurchyshyn, V., Qiu, J., Hickey, J., Montañés Rodriguez, P., Chu, M. C., Kolbe, E., Brown, C. T., \& Koonin, S. E. 2003, J. of Geophys. Res., Vol. 108, No. D22, 4710

Rossow, W. B., Walker, A. W., Beuschel, D. E., \& Roiter, M. D. 1996, World Climate Research Programme Rep. (WMO/TD 737) 